\title{
Investigation of physicochemical composition and microbial communities in traditionally fermented vrum from Inner Mongolia
}

\author{
Yamei,, Yuan-Sheng Guo,, Jian-Jun Zhu, Fang Xiao, Hasiqimuge, Jian-Ping Sun, Jun-Ping Qian, \\ Wei-Liang Xu, Chun-Dong Li, and Liang Guo† \\ Xilingol Vocational College, Xilin Gol Institute of Bioengineering, Xilin Gol Food Testing and Risk Assessment Center, Xilinhot 026000 , \\ Inner Mongolia, China
}

\begin{abstract}
Mongolian traditionally fermented vrum is known for its functional characteristics, and indigenous microbial flora plays a critical role in its natural fermentation. However, studies of traditionally fermented vrum are still rare. In this study, we investigated the artisanal production of traditionally fermented vrum from Inner Mongolia. In general, its physicochemical composition was characterized by $34.5 \pm 8 \%$ moisture, $44.9 \pm 12.1 \%$ fat, $10.6 \pm 3.2 \%$ protein, and $210 \pm 102^{\circ} \mathrm{T}$. The total lactic acid bacteria and yeast counts ranged from 50 to $2.8 \times 10^{8} \mathrm{cfu} / \mathrm{g}$ and from 0 to $1.1 \times 10^{6} \mathrm{cfu} / \mathrm{g}$, respectively. We studied bacterial and fungal community structures in 9 fermented vrum; we identified 5 bacterial phyla represented by 11 genera (an average relative abundance $>1 \%$ ) and 8 species $(>1 \%)$, and 3 fungal phyla represented by 8 genera $(>1 \%)$ and 8 species $(>1 \%)$. Relative abundance values showed that Lactococcus and Lactobacillus were the most common bacterial genera, and Dipodascus was the predominant fungal genus. This scientific investigation of the nutritional components, microbial counts, and community profiles in Mongolian traditionally fermented vrum could help to develop future functional biomaterials and probiotics.

Key words: fermentation, vrum, bacterial, fungal
\end{abstract}

\section{INTRODUCTION}

Traditionally fermented dairy products originated from the spontaneous fermentation of milk or its derivatives using indigenous microflora inhabiting the dairy matrices and the surrounding environment, and they can be traced back to approximately 10,000

Received January 13, 2019.

Accepted June 13, 2019.

*These authors contributed equally to this work.

†Corresponding author: herdman86@163.com
BC (Fuquay, 2011). Fermented dairy products have improved taste, enriched nutrition, enhanced digestibility, and an extended shelf life. Historically, Mongolian nomadic people have created a wide variety of artisanal fermented dairy products, such as Airag, koumiss, Hurood cheese, Tarag, Jueke, and fermented vrum. Traditionally, fermented vrum - a high-fat dairy product from cow milk - comes from a 2-step spontaneous fermentation of original vrum at relatively low temperatures for an extended period in cow or sheep cecum or rumen in Inner Mongolia, China. The manufacturing process for vrum was recorded in an ancient Chinese comprehensive agricultural book called Qi Min Yao Shu, written by Sixie Jia in the 6th century AD.

A flow diagram describing the production of vrum and traditionally fermented vrum can be found in Figure 1. First, filtered cow's milk is heated to the boiling point while stirring constantly to yield as much milk foam as possible (Figure 1A). The milk and foam are kept warm for approximately $10 \mathrm{~h}$ to form the typical honeycomb vrum (Figure 1B). Then, the excess milk and water are drained off and eliminated by evaporation in a clean, well-ventilated area (Figure 1C). Then, the vrum is moved into a container made of wood or porcelain that has a layer of Mongolian traditional cheese at the bottom (Figure 1D). The cheese, which contains natural microbiota, serves as a homemade starter culture for fermentation. Fermentation then occurs spontaneously in summer at ambient temperatures (approximately 18 to $25^{\circ} \mathrm{C}$ ) for 2 to $4 \mathrm{mo}$. The fresh cecum and rumen of cattle or sheep are washed and soaked with sour whey (a byproduct of artisanal cheese production) and spread out to dry; then, they are covered in a layer of artisanal butter to ensure their integrity. The preprocessed cecum and rumen are then filled with the prefermented vrum for spontaneous fermentation in autumn at ambient temperatures (approximately 10 to $20^{\circ} \mathrm{C}$ ) for 2 to 3 mo (Figure $1 \mathrm{E}$ and $1 \mathrm{~F}$ ).

Traditionally fermented vrum is stored sealed in a cool, dry place. On the prairie, with no wooden, metal, or porcelain containers, herdsmen use bovine or ovine 
cecum and rumen to create a relatively closed environment for the natural fermentation of vrum. This ancient craft has provided a foundation for the industrial production of fermented dairy products, and is a precious cultural heritage. The use of bovine or ovine cecum and rumen to ferment milk products such as vrum is a symbol of the back-to-origins ideology of the Mongolian population. Moreover, from the perspective of manufacturing, traditionally fermented vrum is an ancestor of industrial cultured cream: milk is heated to boiling for sterilization, stirred to yield fat-rich foam, and then indigenous microorganisms from traditional cheese or the ruminant digestive tract are applied as a starter culture for spontaneous fermentation. Moreover, only 2 administrative divisions of Inner Mongolia (Hulun Buir and Xilin Gol) maintain the heritage of Mongolian traditionally fermented vrum production, and the number of nomads who have mastered the artisanal skills has dropped dramatically in the past few decades, accompanied by the rapid development of industrialization and urbanization on the prairie.

The time-consuming fermentation and complex manufacturing of vrum have challenged the survival of this traditional product, and production of fermented vrum seems to be in danger of dying out. Traditional production of fermented vrum needs to be investigated and protected. Although fermented vrum is scarce on the prairie, its rich nutrition, distinctive taste, and versatile functions (including detoxification and antiemetic effect) win praise among herdsmen in Inner Mongolia (Shuangquan et al., 2018). However, the study of original and fermented vrum has been limited, and the same or similar products from other species have not been documented or appeared in the market. Previous studies have demonstrated that original vrum, which was a high-fat dairy food with high temperature sterilization, was fermented spontaneously at low temperatures for a long time ( 3 to $5 \mathrm{mo}$ ) to create Mongolian traditionally fermented vrum (Zhao and Li, 2007; Shuangquan et al., 2018). In addition, the microbial flora involved in fermenting of vrum at low temperatures for a long time are new territory for the research and development of indigenous probiotics. In general, traditionally fermented dairy products are a major source for screening and isolating beneficial microorganisms such as Lactobacillus helveticus NS8 (Rong et al., 2015), Lactobacillus plantarum JLK0142 (Wang et al., 2018a), Kluyveromyces marxianus, and Kluyveromyces lactis (Valderrama et al., 1999). However, the nutritional and microbial composition of traditionally fermented vrum has not yet been investigated in detail.

This study aimed to investigate the traditional production of artisanal fermented vrum to record this valuable cultural product and assess its nutritional value. We also aimed to analyze the bacterial and fungal community profiles and predominant microbes using culture-dependent methods and 16S rRNA and internal transcribed spacer (ITS) amplicon sequencing. This microbial investigation will provide fundamental information for understanding the spontaneous fermentation of traditionally fermented vrum, and for screening and isolating future probiotics and functional biomaterials.

\section{MATERIALS AND METHODS}

\section{Collection of Traditionally Fermented Vrum}

We collected 9 samples of artisanal fermented vrum from the Mongolian nomadic people in Inner Mongolia, including 7 samples (FV1, FV2, FV3, FV4, FV5, FV6,

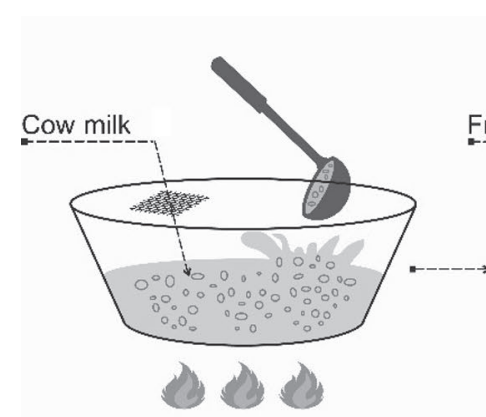

(A)

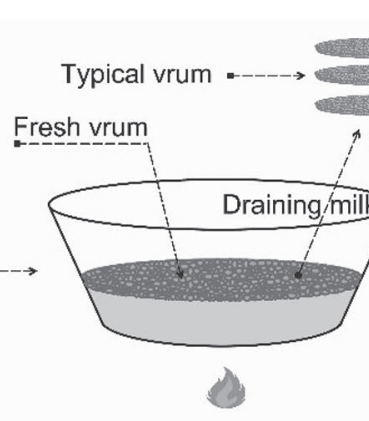

(B)

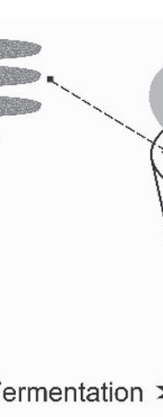

Fermentation >

Figure 1. Flow diagram for production of Mongolian traditionally fermented vrum. (A) Filtered cow's milk is heated to the boiling point and stirred to create foam. (B) Honeycomb fresh vrum is formed on top by keeping the milk warm. (C) Excess milk and water are drained and eliminated by evaporation. (D) Vrum is pre-fermented in a wooden or porcelain barrel using Mongolian artisanal cheese as a culture starter. The cecum (E) and rumen (F) are filled with the pre-fermented vrum to produce traditionally fermented vrum via spontaneous fermentation in autumn for 2 to 3 mo at ambient temperatures. 
and FV7) from Hulun Buir and 2 (FV8 and FV9) from Xilin Gol. These samples were immediately stored at $4^{\circ} \mathrm{C}$ and analyzed to investigate physicochemical composition, enumerate microorganisms, and create bacterial and fungal community profiles.

\section{Measurement of Physicochemical Indexes and Enumeration of Microorganisms}

We measured the moisture (China National Food Safety Standard, 2016a), fat (China National Food Safety Standard, 2016b), protein (China National Food Safety Standard, 2016c), lactose (China National Food Safety Standard, 2010a), acidity (China National Food Safety Standard, 2016h), and fatty acid (China National Food Safety Standard, 2016d) content of the vrum according to China's national food safety standards. We determined fat content using the Soxhlet extraction method (China National Food Safety Standard, 2016b) and protein content using the Kjeldahl method (China National Food Safety Standard, 2016c). We calculated lactose content using titration with Feline's solution and methylene blue. We measured the acidity of fermented vrum by titration with phenolphthalein indicator. We enumerated lactic acid bacteria (LAB; China National Food Safety Standard, 2016e) counts on de Man, Rogosa and Sharpe agar for $72 \mathrm{~h}$ at $36^{\circ} \mathrm{C}$ according to China's national food safety standards. We enumerated yeast and mold (China National Food Safety Standard, 2016f) counts using Rose Bengal agar for $5 \mathrm{~d}$ at $28^{\circ} \mathrm{C}$. We determined coliform counts (China National Food Safety Standard, 2016g) using Violet Red Bile Agar and Brilliant Green Lactose Bile for 48 $\mathrm{h}$ at $36^{\circ} \mathrm{C}$.

\section{PCR Amplification and Amplicon Sequencing}

We isolated microbial DNA from traditionally fermented vrum using an E.Z.N.A stool DNA kit (Omega Bio-tek, Norcross, GA) according to the manufacturer's protocol in the kit. We amplified the 16S rRNA V3-V4 region of the ribosomal RNA gene by PCR using primers 341F 5'-CCTACGGGNGGCWGCAG-3' and 806R 5'-GGACTACHVGGGTATCTAAT-3'. We amplified the ITS region of the ribosomal RNA gene using primers ITS3-KYO2F 5'-GATGAAGAACGYAGYRAA-3' and ITS4R 5'-TCCTCCGCTTATTGATATGC-3'. The PCR reaction (running program: $95^{\circ} \mathrm{C}$ for $2 \mathrm{~min}$; 27 cycles of $98^{\circ} \mathrm{C}$ for $10 \mathrm{~s}, 62^{\circ} \mathrm{C}$ for $30 \mathrm{~s}$, and $68^{\circ} \mathrm{C}$ for 30 $\mathrm{s} ; 68^{\circ} \mathrm{C}$ for $10 \mathrm{~min}$ ) was executed in a tube containing $5 \mu \mathrm{L}$ of $10 \times$ KOD buffer, $5 \mu \mathrm{L}$ of $2.5 \mathrm{~m} M$ dNTPs, 1.5 $\mu \mathrm{L}$ of each primer $(5 \mu M), 1 \mu \mathrm{L}$ of KOD polymerase, and $100 \mathrm{ng}$ of template DNA isolated from traditionally fermented vrum. The 16S rRNA and ITS amplicons were isolated from $2 \%$ agarose gel and purified using an AxyPrep DNA Gel Extraction Kit (Axygen Biosciences, Union City, CA). These amplicons were quantified and paired-end sequenced $(2 \times 250)$ using the Illumina platform (Illumina Inc., San Diego, CA).

\section{Bioinformatics and Statistical Analysis}

Raw reads were filtered to obtain high-quality clean reads by removing the reads containing more than $10 \%$ unknown nucleotides and less than $80 \%$ of bases with a quality (Q-value) of less than 20. Clean tags were searched in the reference database to perform chimera checking using the UCHIME algorithm. After we removed chimeric tags, we obtained the effective tags. These effective tags were clustered into operational taxonomic units (OTU) of $\geq 97 \%$ similarity using the UPARSE pipeline (Edgar, 2013). The OTU were classified into organisms using the Naive Bayesian Model and RDP classifier (Wang et al., 2007) based on the SILVA database for 16S rRNA (Pruesse et al., 2007) and the UNITE database for ITS (Kõljalg et al., 2005). The rarefaction curve, Chao1, Shannon, Simpson, and Good's coverage were determined in QIIME (Caporaso et al., 2010).

\section{RESULTS AND DISCUSSION}

\section{Nutritional and Microbiological Analyses of Fermented Vrum}

Mongolian artisanal fermented vrum is a highly nutritional dairy product that is rich in fat and protein. In the samples we evaluated, the fat content [mean \pm standard deviation (SD)] was $44.9 \pm 12.1 \%$, and the protein content was $10.6 \pm 3.2 \%$ (Figure 2 and Table 1). Fermented vrum is derived from the spontaneous fermentation of vrum, and indigenous microorganisms that metabolize lactose into lactic acid play an essential role in spontaneous fermentation. The lactose content (mean $\pm \mathrm{SD}$ ) of fermented vrum was $4.93 \pm 3.07 \%$, and its acidity was $210 \pm 102^{\circ} \mathrm{T}$ (Figure 2 and Table 1 ). Traditionally fermented vrum is similar in fat content to cultured cream, which ranges in fat content from $10 \%$ to more than 40\% (Fuquay, 2011). The acidification degree of fermented vrum was higher than that of sour cream (Huang et al., 2016) and fermented milk (China National Food Safety Standard. 2010b). Compared to the reported contents of vrum (Xiao, 2013), the amount of fat decreased from vrum to fermented vrum, and protein content remained unchanged during fermentation. We speculate that the fat in fermented 

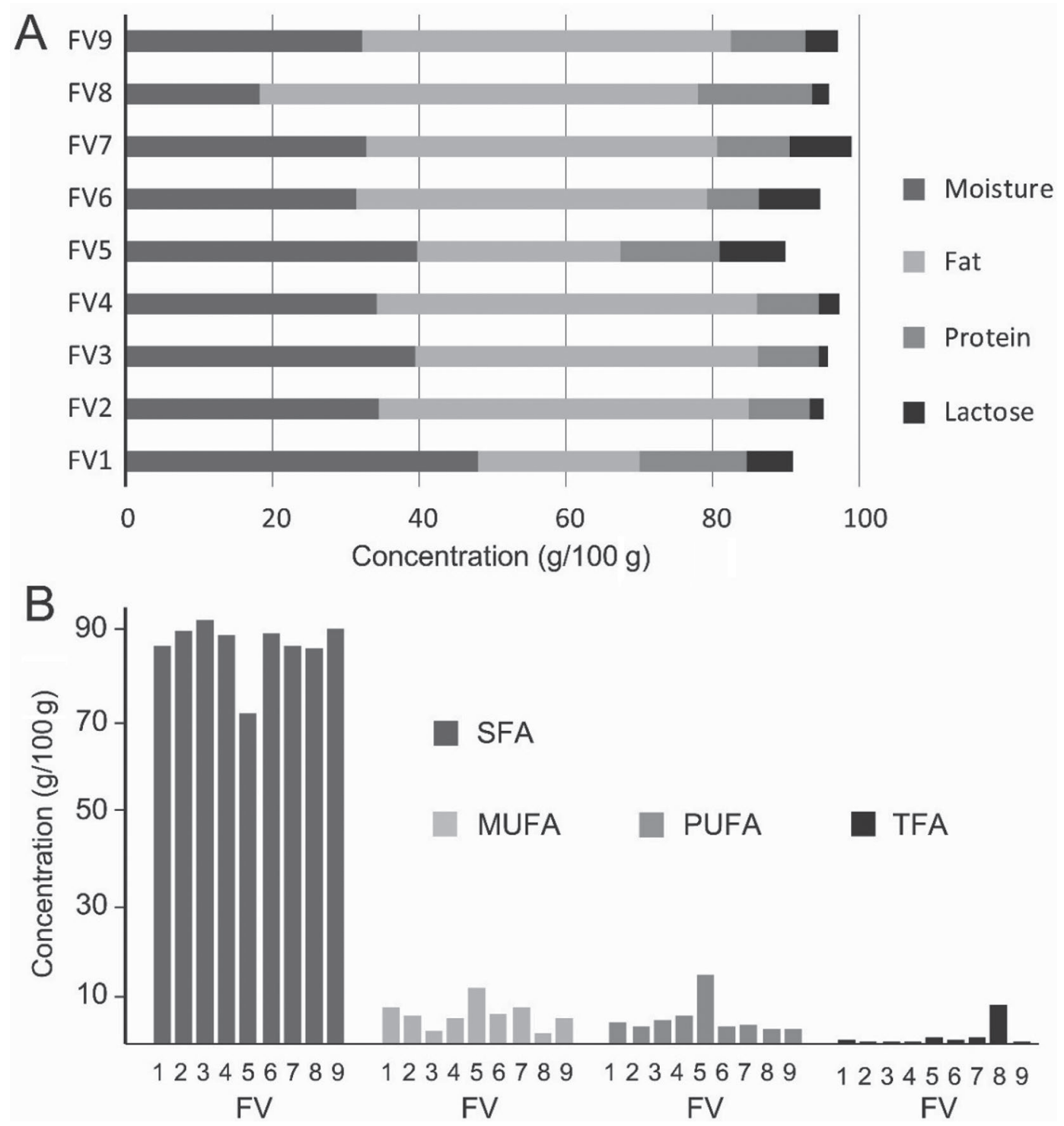

Figure 2. Physicochemical composition of 9 fermented vrum from Inner Mongolia. (A) We measured moisture, fat, protein, and lactose content according to China's national food safety standards. (B) The percentages of different fatty acids in the 9 fermented vrum (by sample number) are shown in a histogram. TFA = trans fatty acid; $\mathrm{FV}=$ fermented vrum.

vrum is metabolized to fatty acids by microorganisms. As shown in Figure 2 and Table 1, the fatty acid contents (mean $\pm \mathrm{SD}$ ) in the fat fraction were $86.97 \pm$ $6 \%$ for SFA, $6.17 \pm 2.92 \%$ for MUFA, $5.33 \pm 3.74 \%$ for PUFA, and $1.48 \pm 2.65 \%$ for trans fatty acid. The most abundant SFA, MUFA, and PUFA in fermented vrum were palmitic acid (C16:0), oleic acid (C18:1), and linoleic acid (C18:2), respectively. Fermented vrum is derived from cow's milk, but we found that the SFA content in fermented vrum exceeded that of milk (68.72\%; Markiewicz-Keszycka et al., 2013). The decomposition of fat likely led to an increase in SFA content during spontaneous fermentation.
The microbiota in fermented vrum play an essential role in spontaneous fermentation. The total LAB count ranged from $2.9 \times 10^{3}$ to $2.8 \times 10^{8} \mathrm{cfu} / \mathrm{g}$, but FV8 contained only $50 \mathrm{cfu} / \mathrm{g}$ (Table 2 ). The LAB are closely related to various habitats, particularly those rich in nutritional components such as dairy matrices, where they generate lactic acid and some carbon dioxide from lactose during fermentation. In a previous study, the total LAB count varied from 3.41 to $9.03 \log \mathrm{cfu} / \mathrm{mL}$ in traditionally fermented dairy products in Mongolia ( $\mathrm{Yu}$ et al., 2011). We found greater variations in total LAB count in traditionally fermented vrum, and the total LAB count in FV8 was only $50 \mathrm{cfu} / \mathrm{g}$. The relationship 
Table 1. Physicochemical composition of traditionally fermented vrum from Inner Mongolia

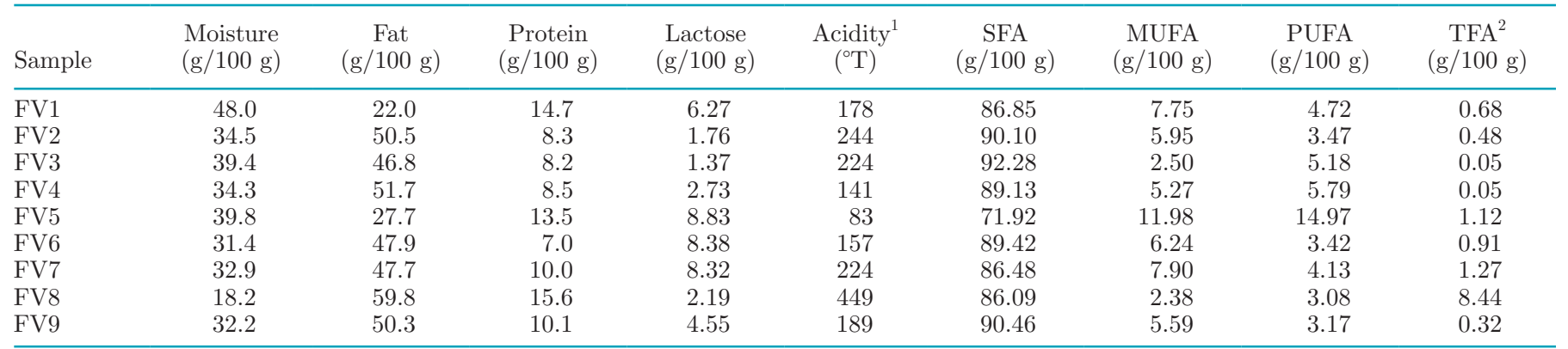

${ }^{1}$ Degrees Theurer.

${ }^{2} \mathrm{TFA}=$ trans fatty acid.

between acidity and LAB suggests that high acidity could inhibit the reproduction of LAB in FV8 (acidity: $\left.449^{\circ} \mathrm{T}\right)$. Traditionally fermented dairy products such as Hurood cheese, Jueke, Tarag, and cheese are made by mixed microflora, which contain various LAB and yeasts (Sun et al., 2014; Gao et al., 2017; Murugesan et al., 2018). As shown in Table 2, the yeast count ranged from 60 to $1.1 \times 10^{6} \mathrm{cfu} / \mathrm{g}$, and none of viable yeast had been cultured from FV3 and FV8. Moreover, along with LAB and yeasts, molds and coliforms are often found in traditional dairy products, and likely to spoil the dairy matrix (Martin et al., 2016; Garnier et al., 2017). The mold count in the samples we examined ranged from 10 to $3.2 \times 10^{3} \mathrm{cfu} / \mathrm{g}$ (Table 2 ). We found no trace of coliform, and we perceived this to be an indicator of the health standard of the food; a high coliform count can lead to deterioration in the quality of dairy products. We speculate that the high acidity of these acidified fermented vrum could inhibit the growth of coliforms.

\section{Bacterial Sequence Abundance and Community Profile of Fermented Vrum}

We characterized the fermented vrum samples from Inner Mongolia by $16 \mathrm{~S}$ rRNA amplicon sequencing.
Following sequence quality control, we obtained a total of 581,214 bacterial high-quality sequences, with an average of 64,579 [standard deviation (SD) 7,544] sequences for each fermented vrum. The average length of the $16 \mathrm{~S}$ rRNA amplicons was 423 (SD 4). The highquality bacterial sequences were clustered into OTU of $\geq 97 \%$ similarity, and we identified a total of 612 bacterial OTU from the 9 samples; the number of OTU per sample ranged from 31 to 92 (Table 3 ). We assessed the bacterial community richness and diversity for each fermented vrum using Chao1, Shannon, Simpson, and Good's coverage (Table 3). Good's coverage was $>99 \%$, indicating that the bacterial community was adequately represented. The Shannon diversity curves achieved the saturation phase from the analysis of bacterial community diversity, but not the rarefaction curves (Figure 3A and $3 \mathrm{~B}$ ). These results show that the majority of bacterial phylotypes had already been obtained, although some new bacterial phylotypes could be characterized by increasing the depth of amplicon sequencing.

We analyzed bacterial relative abundance at the phylum level, and identified 5 phyla. The bacterial community consisted mainly of Firmicutes (relative abundance $75.27 \%)$, Proteobacteria (23.69\%), Cyanobacteria (0.95\%), Bacteroidetes (0.06\%), and Actinobacteria $(0.02 \%)$, but Firmicutes dominated in all the samples.

Table 2. Microbial counts of traditionally fermented vrum from Inner Mongolia ${ }^{1}$

\begin{tabular}{lcccc}
\hline Sample & LAB $(\mathrm{cfu} / \mathrm{g})$ & Yeast $(\mathrm{cfu} / \mathrm{g})$ & Mold $(\mathrm{cfu} / \mathrm{g})$ & Coliform $(\mathrm{cfu} / \mathrm{g})$ \\
\hline FV1 & $2.8 \times 10^{8}$ & $1.1 \times 10^{6}$ & $3.2 \times 10^{3}$ & $\mathrm{ND}^{2}$ \\
FV2 & $1.9 \times 10^{7}$ & $2.3 \times 10^{5}$ & 10 & ND \\
FV3 & $6.1 \times 10^{5}$ & $\mathrm{ND}$ & 10 & $\mathrm{ND}$ \\
FV4 & $1.7 \times 10^{7}$ & $1.3 \times 10^{4}$ & $6.8 \times 10^{2}$ & $\mathrm{ND}$ \\
FV5 & $2.9 \times 10^{3}$ & $3.6 \times 10^{3}$ & $1.8 \times 10^{2}$ & $\mathrm{ND}$ \\
FV6 & $6.3 \times 10^{6}$ & $2.8 \times 10^{2}$ & $2.8 \times 10^{2}$ & $\mathrm{ND}$ \\
FV7 & $1.8 \times 10^{7}$ & 60 & 20 & $\mathrm{ND}$ \\
FV8 & 50 & $\mathrm{ND}$ & 20 & $\mathrm{ND}$ \\
FV9 & $2.9 \times 10^{8}$ & & & \\
${ }^{1} \mathrm{LAB}=$ lactic acid bacteria. & & &
\end{tabular}


Table 3. Diversity indices from amplicon sequencing of traditionally fermented vrum from Inner Mongolia

\begin{tabular}{|c|c|c|c|c|c|c|c|}
\hline Target & Sample & $\begin{array}{l}\text { No. of } \\
\text { reads }\end{array}$ & $\begin{array}{l}\text { No. of } \\
\text { OTU }^{1}\end{array}$ & $\begin{array}{l}\text { Chao1 } \\
\text { index }\end{array}$ & $\begin{array}{c}\text { Shannon } \\
\text { index }\end{array}$ & $\begin{array}{l}\text { Simpson } \\
\text { index }\end{array}$ & $\begin{array}{l}\text { Good's } \\
\text { coverage }\end{array}$ \\
\hline \multirow[t]{6}{*}{ Bacterial analysis } & FV1 & 70,566 & 76 & 87 & 2.20 & 0.15 & 0.9998 \\
\hline & FV2 & 68,638 & 71 & 84 & 1.84 & 0.23 & 0.9998 \\
\hline & FV4 & 71,453 & 78 & 86 & 2.13 & 0.20 & 0.9998 \\
\hline & FV5 & 51,018 & 92 & 110 & 1.15 & 0.55 & 0.9997 \\
\hline & FV6 & 64,465 & 64 & 72 & 2.24 & 0.17 & 0.9998 \\
\hline & FV9 & 74,930 & 41 & 44 & 1.48 & 0.32 & 0.9999 \\
\hline \multirow[t]{6}{*}{ Fungal analysis } & FV1 & 8,304 & 64 & 85 & 1.14 & 0.37 & 0.9998 \\
\hline & FV2 & 13,340 & 74 & 110 & 1.74 & 0.50 & 0.9999 \\
\hline & FV3 & 12,307 & 79 & 94 & 2.16 & 0.70 & 0.9998 \\
\hline & FV4 & 13,507 & 93 & 100 & 3.68 & 0.85 & 0.9999 \\
\hline & FV5 & 16,313 & 110 & 118 & 2.99 & 0.76 & 0.9999 \\
\hline & FV6 & 8,272 & 62 & 75 & 0.92 & 0.30 & 0.9999 \\
\hline
\end{tabular}

${ }^{1}$ Operational taxonomic units.

Our findings were similar to those of previous studies, and showed the 4 major bacterial phyla (Firmicutes, Proteobacteria, Actinobacteria, Bacteroidetes) and the predominance of Firmicutes in traditionally fermented dairy products (Sun et al., 2014; Gesudu et al., 2016). As well, 7 bacterial orders had an average relative abundance $>1 \%$ : Lactobacillales (68.78\%), Enterobacteriales (11.5\%), Rhodospirillales (7.39\%), Bacillales (6.28\%), Pseudomonadales (2.14\%), Rickettsiales (1.47\%), and Clostridiales (1.23\%).

As shown in Figure 3C, we further analyzed bacterial relative abundance at the genus level. Eleven genera had an average relative abundance $>1 \%$ : Lactococcus $(30.12 \%)$, Lactobacillus (28.56\%), Streptococcus (8.61\%), Acetobacter (7.39\%), Enterobacter (6.28\%), Anoxybacillus (5.37\%), Erwinia (2.28\%), Proteus (1.46\%), Serratia (1.36\%), Pseudomonas (1.1\%), and Acinetobacter (1.01\%). Lactobacillus and Lactococcus $(58.68 \%)$ predominated overall in the bacterial genera and are representative LAB that play an essential role in the nutritional and organoleptic properties of fermented dairy products (Caplice and Fitzgerald,1999; $\mathrm{Yu}$ et al., 2011). Of the 11 bacteria genera, Lactococcus dominated in FV4 and FV9, corresponding to $66.24 \%$ and $67.98 \%$ relative abundance, respectively. Lactobacillus was the predominant bacterial genus in FV1 and FV8, corresponding to $80.07 \%$ and $78.16 \%$ relative abundance, respectively. Lactococcus (40.44\%) and Lactobacillus (29.51\%) predominated in FV3. Previous studies have also demonstrated that Lactococcus and Lactobacillus were the predominant bacterial genera in fermented dairy products (Yu et al., 2011; Lusk et al., 2012; Sun et al., 2014; Gao et al., 2017). Streptococcus was the predominant bacterial genus in FV5, with a relative abundance of $73.57 \%$. Some of the bacterial genera we identified were commercially significant for the fermentation of dairy products (Krzyściak et al., 2013; Li et al., 2016; Khanal and Lucey, 2018). Acetobacter, Lactococcus, and Lactobacillus dominated in FV2, at $48.51,20.83$, and $29.58 \%$ relative abundance, respectively. Acetobacter was able to transform alcohol produced by yeast in fermented vrum via alcohol dehydrogenase to oxidize ethanol to acetic acid (Tian et al., 2017). Lactococcus, Enterobacter, and Lactobacillus dominated in FV7, corresponding to $27.04 \%$, $22.18 \%$, and $14.64 \%$ relative abundance, respectively. Lactococcus and Erwinia dominated in FV6, corresponding to $47.5 \%$ and $11.19 \%$ relative abundance, respectively. Enterobacter and Erwinia were genera of Enterobacteriaceae that have been associated with threats to health and spoilage of foods (Blackburn, 2006; Perez and Bonomo, 2019).

We identified 8 bacterial species, including Lactococcus lactis (40.65\%), Lactobacillus kefiranofaciens (16.98\%), Streptococcus salivarius (11.27\%), Lactobacillus helveticus $(7.63 \%)$, Acetobacter malorum (6.6\%), Lactobacillus kefiri (4.83\%), Acetobacter orientalis (1.69\%), and Lactobacillus diolivorans (1.65\%). Lactococcus lactis dominated in 4 fermented vrum (FV4, FV6, FV7, and FV9), corresponding to $71.57 \%, 77.52 \%, 54.55 \%$, and $91.51 \%$ relative abundance, respectively. Lactococcus lactis and Lactobacillus helveticus dominated in FV3, corresponding to $48.38 \%$ and $24.36 \%$ relative abundance, respectively. Acetobacter malorum and Lactococcus lactis dominated in FV2, corresponding to $41.91 \%$ and $21.06 \%$ relative abundance, respectively. Streptococcus 
salivarius and Lactobacillus kefiranofaciens dominated in FV5 (95.09\%) and FV8 (87.71\%), respectively. Lactobacillus kefiri and Lactobacillus kefiranofaciens dominated in FV1, corresponding to $32.87 \%$ and $28.59 \%$ relative abundance, respectively. Lactococcus lactis and
Lactobacillus helveticus have been used extensively in the production of fermented products (Kelly et al., 2010; Slattery et al., 2010; Cavanagh et al., 2015; Sun et al., 2015). Lactobacillus kefiranofaciens and Lactobacillus kefiri, which have been isolated from kefir grains,
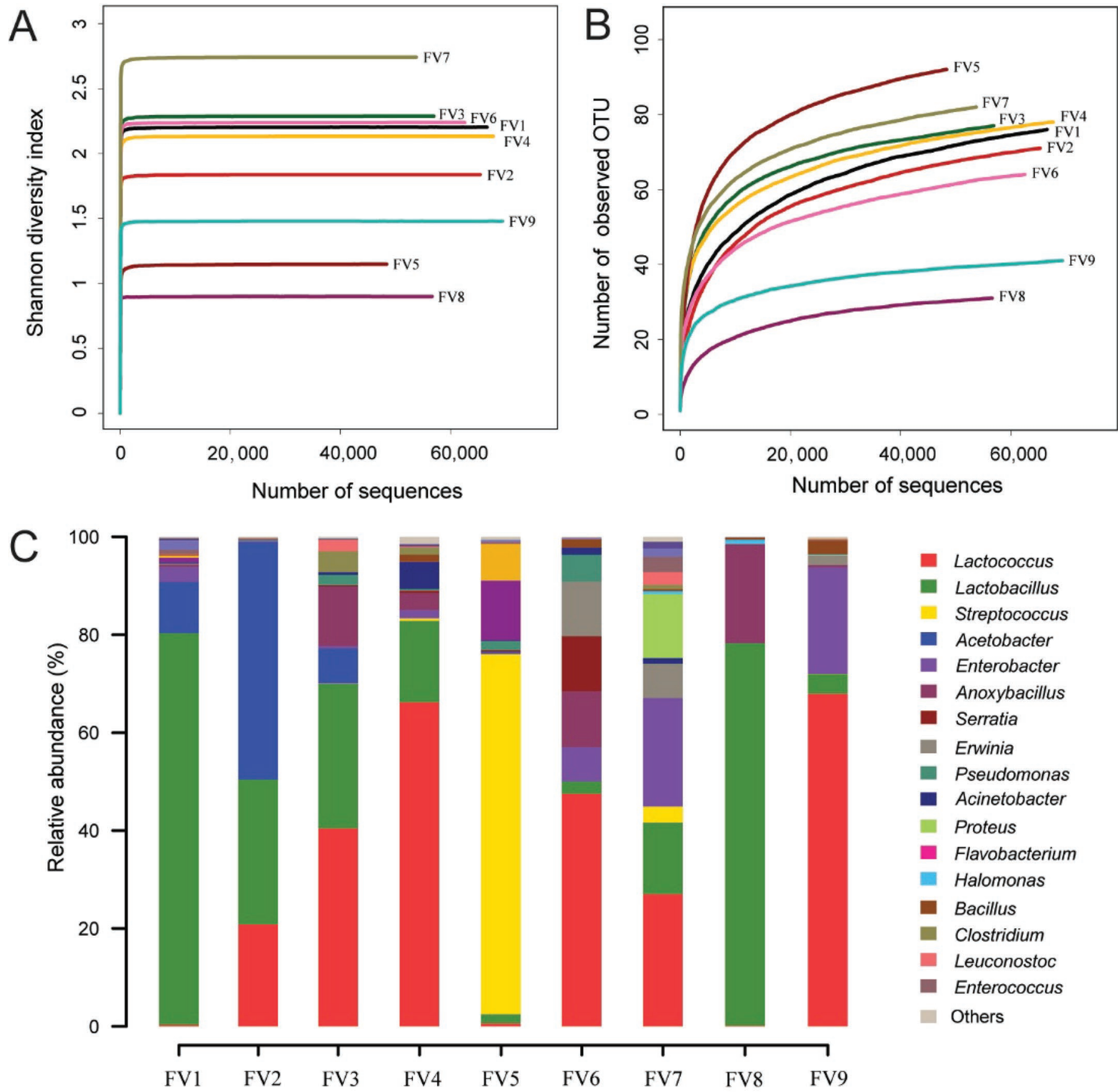

Figure 3. (A) Shannon diversity curves and (B) rarefaction curves for 16S rRNA sequencing analysis. (C) Relative abundance of bacterial sequences at the genus level in 9 fermented vrum (FV) from Inner Mongolia. OTU = operational taxonomic units. 

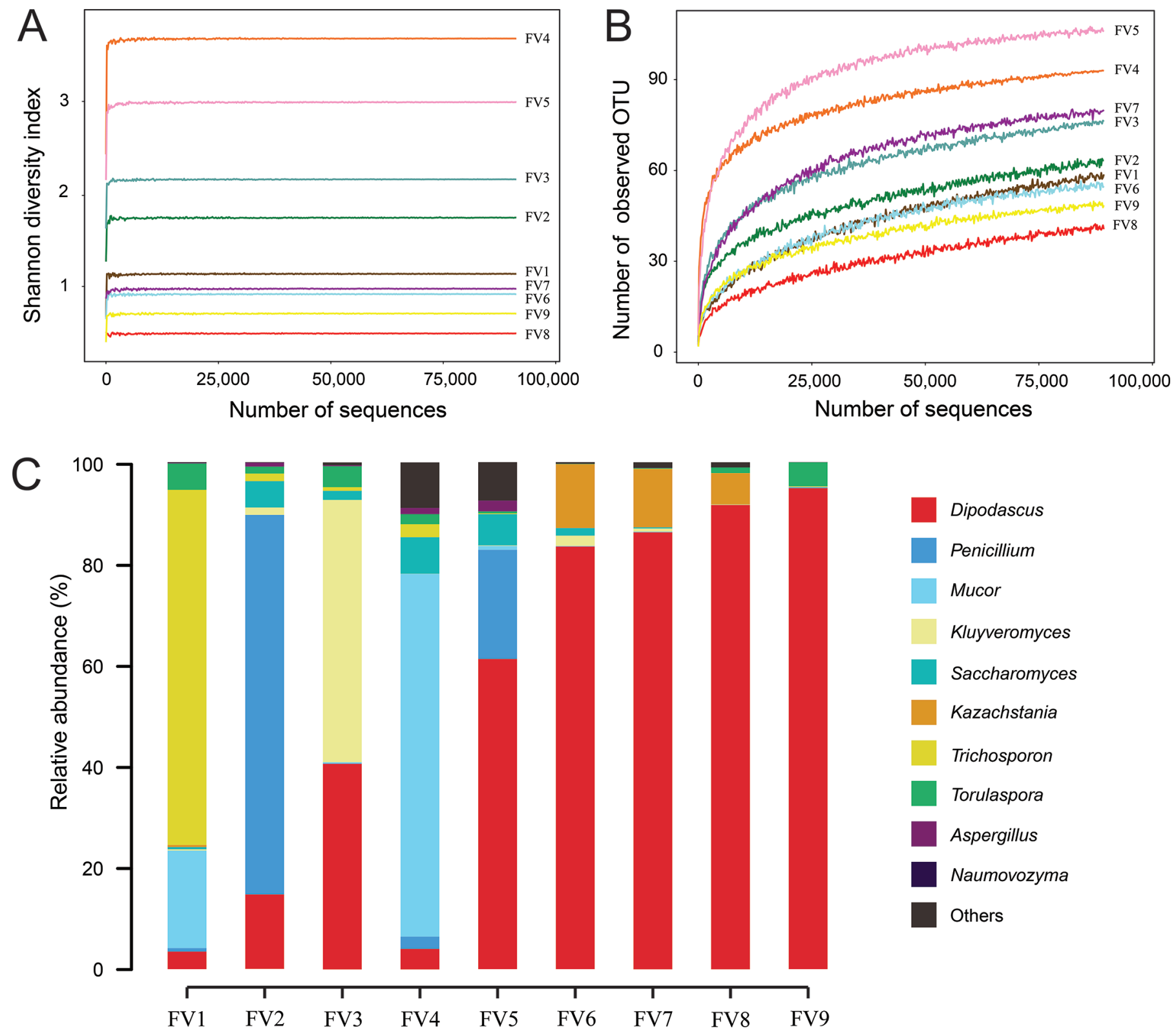

Figure 4. (A) Shannon diversity curves and (B) rarefaction curves for internal transcribed spacer sequencing analysis. (C) Relative abundance of fungal sequences at the genus level in 9 fermented vrum (FV) from Inner Mongolia. OTU = operational taxonomic units.

exhibit probiotic and antimicrobial attributes (Carasi et al., 2014; Xing et al., 2017; Federici et al., 2018; Jeong et al., 2018; Wang et al., 2018b).

\section{Fungal Sequence Abundance and Community Profile of Fermented Vrum}

We analyzed the fungal community profile using ITS amplicon sequencing. We generated a total of 93,706 high-quality fungal ITS sequences from 9 fermented vrum samples, with an average of 10,412 (SD 3,518) sequences for each fermented vrum. The average length of ITS amplicons was 344 (SD 22). These sequences were clustered into 668 fungal OTU (ranging from 48 to 110) (Table 3). Based on the analysis of $\alpha$ diversity for the fungal sequences from the 9 fermented vrum, the Shannon diversity curves reached the saturation phase (Figure 4A), but the rarefaction curves did not (Figure $4 \mathrm{~B})$. These results showed that the majority of fungal phylotypes for fermented vrum have already been cap- 
tured in analysis, although some additional new fungal phylotypes could be identified by increasing the depth of sequencing of the ITS amplicon. Good's coverage (values $>99 \%$ ) suggested that the majority of fungal phylotypes obtained from fermented vrum had already been captured (Table 3).

In the bioinformatics analysis of sequences, we used Chao1 analysis to account for the bacterial and fungal richness of fermented vrum, and the Shannon and Simpson indexes to explain the diversity of bacteria and fungi in fermented vrum (Table 3). Our findings showed that the Chaol index of bacteria $(78 \pm 25)$ was lower than that of fungi $(91 \pm 17)$ in fermented $\operatorname{vrum}(P<0.05)$, and we found no real distinction in the Shannon and Simpson indexes between the bacterial and the fungal diversity $(P>0.01)$. The above comparative $\alpha$ indexes demonstrated that traditionally fermented vrum had more fungal phylotypes than bacterial phylotypes, and fungal diversity was similar to bacterial diversity.

From the sequencing of the ITS amplicon, we identified 3 phyla: Ascomycota (relative abundance: 89.44\%), Mucoromycota (8.27\%), and Basidiomycota (2.29\%): Ascomycota dominated in all samples but FV4, in which the predominant phylum was Mucoromycota (67.91\%). In previous studies, the predominant phylum identified in traditional dairy products was Ascomycota (Sun et al., 2014; Gao et al., 2017). Four other fungal orders had an average relative abundance $>1 \%$ : Saccharomycetales (76.75\%), Eurotiales (11.01\%), Mucorales $(8.27 \%)$, and Trichosporonales $(2.07 \%)$.

As shown in Figure 4C, we identified 8 fungal genera with an average relative abundance $>1 \%$ : Dipodascus (53.33\%), Penicillium (11.1\%), Mucor (10.22\%), Trichosporon (8.34\%), Kluyveromyces (6.26\%), Kazachstania $(3.42 \%)$, Saccharomyces (2.51\%), and Torulaspora $(2.12 \%)$. Dipodascus dominated in 5 samples (FV5, FV6, FV7, FV8, and FV9), corresponding to $61.19 \%$, $83.35 \%, 86.2 \%, 91.58 \%$ and $94.87 \%$ relative abundance, respectively. Kluyveromyces and Trichosporon dominated in FV3 (51.74\%) and FV1 (70.04\%). Most fungi in the 7 samples belonged to yeast, and have previously been identified in traditional dairy products (Sun et al., 2014; Gao et al., 2017). However, Dipodascus has not been previously reported in dairy foods. The relative abundance findings showed that Penicillium (74.9\%) and Mucor $(71.56 \%)$ were the predominant genera in FV2 and FV4, respectively. In previous research, these 2 genera have been described as filamentous fungi that can bring about dairy spoilage such as off-odor and dairy waste (Garnier et al., 2017). Based on our knowledge of dairy fermentation, the occurrence of spoilage fungi in fermented vrum suggests that spontaneous fermentation could be contaminated by detrimental microorganisms that originate from raw materials, equipment, tools, and unsanitary environment. However, these filamentous fungi may contribute to the distinct flavor of Mongolian artisanal fermented vrum.

We identified 8 fungal species: Kazachstania unispora (29.5\%), Saccharomyces cerevisiae (16.1\%), Trichosporon asahii (13.9\%), Kluyveromyces marxianus (12.2\%), Mucor racemosus (8.63\%), Kluyveromyces lactis (6.02\%), Pichia fermentans (4.46\%), and Mucor circinelloides (3.61\%). Similar to our analysis of the bacterial community, the varieties and relative abundance of fungal species were obviously distinct in individual fermented vrum. Such variation in fungal diversity was also discovered in spontaneously fermented cow's milk from different geographic locations (Sun et al., 2014).

\section{CONCLUSIONS}

Traditionally fermented vrum, which has a long history in Inner Mongolia, receives praise for its nutritional profile and versatile functions. In this study, we investigated the classic manufacture of artisanal fermented vrum and illustrated the process using flow diagrams. Traditionally fermented vrum is the ancestor of industrial sour cream, from the perspective of manufacturing processes and nutritional profile. Our investigation of the physicochemical and microbial composition of traditionally fermented vrum demonstrated that it was a fatty sour dairy food rich in LAB and yeast. Finally, our results identified 5 bacterial phyla represented by 11 genera and 8 species, and 3 fungal phyla represented by 8 genera and 8 species. Lactococcus and Lactobacillus dominated at the level of bacterial genus, and Dipodascus was the predominant fungal genus. The varieties and abundances of the bacterial and fungal genera were different among the 9 samples of fermented vrum we evaluated. We speculate that the microbiological characteristics of raw vrum, the natural starter culture from Mongolian traditional cheese, and environmental and tool sanitation were all likely to influence the microbial community structure of traditionally fermented vrum.

\section{ACKNOWLEDGMENTS}

We thank Miao-Miao Hao, Yun-Fang Wu, and Nanslama for the nutritional and microbiological experiments. We thank Yan Ju for providing some related references. Funding was provided by Xilingol Vocational College Key Research Project (ZD-2019-06 and ZD-2019-10), Inner Mongolia College Science Research Project (NJZY19336) and Chinese National Key Research and Development Program (2017YFE0108800). 


\section{REFERENCES}

Blackburn, C. de W. 2006. Food Spoilage Microorganisms. Woodhead Publishing, Cambridge, United Kingdom.

Caplice, E., and G. F. Fitzgerald. 1999. Food fermentations: Role of microorganisms in food production and preservation. Int. J. Food Microbiol. 50:131-149.

Caporaso, J. G., J. Kuczynski, J. Stombaugh, K. Bittinger, F. D. Bushman, E. K. Costello, N. Fierer, A. G. Pena, J. K. Goodrich, J. I. Gordon, G. A. Huttley, S. T. Kelley, D. Knights, J. E. Koenig, R. E. Ley, C. A. Lozupone, D. McDonald, B. D. Muegge, M. Pirrung, J. Reeder, J. R. Sevinsky, P. J. Turnbaugh, W. A. Walters, J. Widmann, T. Yatsunenko, J. Zaneveld, and R. Knight. 2010. QIIME allows analysis of high-throughput community sequencing data. Nat. Methods 7:335-336.

Carasi, P., M. Diaz, S. M. Racedo, G. De Antoni, M. C. Urdaci, and L. Serradell Mde. 2014. Safety characterization and antimicrobial properties of kefir-isolated Lactobacillus kefiri. BioMed Res. Int. 2014:208974.

Cavanagh, D., G. F. Fitzgerald, and O. McAuliffe. 2015. From field to fermentation: the origins of Lactococcus lactis and its domestication to the dairy environment. Food Microbiol. 47:45-61.

China National Food Safety Standard. 2010a. Determination of lactose and sucrose in foods for infants and young children. GB 5413.52010. Ministry of Health of the People's Republic of China, China.

China National Food Safety Standard. 2010b. National food safety standard fermented milk. GB 19302-2010. Ministry of Health of the People's Republic of China, China.

China National Food Safety Standard. 2016a. Determination of moisture in foods. GB 5009.3-2016. Ministry of Health of the People's Republic of China, China.

China National Food Safety Standard. 2016b. Determination of fat in foods. GB 5009.6-2016. Ministry of Health of the People's Republic of China, China.

China National Food Safety Standard. 2016c. Determination of protein in foods. GB 5009.5-2016. Ministry of Health of the People's Republic of China, China.

China National Food Safety Standard. 2016d. Determination of fatty acids in foods. GB 5009.168-2016. Ministry of Health of the People's Republic of China, China.

China National Food Safety Standard. 2016e. Detection of food microorganisms - lactic acid bacteria. GB4789.35-2016. Ministry of Health of the People's Republic of China, China.

China National Food Safety Standard. 2016f. Detection of food microorganisms - yeast and mold. GB4789.15-2016. Ministry of Health of the People's Republic of China, China.

China National Food Safety Standard. 2016g. Detection of food microorganisms - coliform. GB4789.3-2016. Ministry of Health of the People's Republic of China, China.

China National Food Safety Standard. 2016h. Determination of acidity in foods. GB 5009.239-2016. Ministry of Health of the People's Republic of China, China.

Edgar, R. C. 2013. UPARSE: Highly accurate OTU sequences from microbial amplicon reads. Nat. Methods 10:996-998.

Federici, F., L. Manna, E. Rizzi, E. Galantini, and U. Marini. 2018. Draft genome sequence of Lactobacillus kefiri SGL 13, a potential probiotic strain isolated from kefir grains. Microbiol. Resour. Announc. 7:e00937-18.

Fuquay, J. W. 2011. Encyclopedia of Dairy Sciences. Elsevier, Amsterdam, the Netherlands.

Gao, M. L., H. M. Hou, X. X. Teng, Y. L. Zhu, H. S. Hao, and G. L. Zhang. 2017. Microbial diversity in raw milk and traditional fermented dairy products (Hurood cheese and Jueke) from Inner Mongolia, China. Genet. Mol. Res. 16:gmr16019451.

Garnier, L., F. Valence, and J. Mounier. 2017. Diversity and control of spoilage fungi in dairy products: An update. Microorganisms 5:E42.

Gesudu, Q., Y. Zheng, X. Xi, Q. C. Hou, H. Xu, W. Huang, H. Zhang, B. Menghe, and W. Liu. 2016. Investigating bacterial population structure and dynamics in traditional koumiss from Inner Mon- golia using single molecule real-time sequencing. J. Dairy Sci 99:7852-7863.

Huang, M. K., Z. C. Li, Y. F. Luo, P. Wang, Y. B. Tang, and H. L. Xie. 2016. Study on preparation technology of fermented butter. China Dairy Indus. 44:57-60. (in Chinese).

Jeong, D., D. H. Kim, K. Y. Song, and K. H. Seo. 2018. Antimicrobial and anti-biofilm activities of Lactobacillus kefiranofaciens DD2 against oral pathogens. J. Oral Microbiol. 10:1472985.

Kelly, W. J., L. J. Ward, and S. C. Leahy. 2010. Chromosomal diversity in Lactococcus lactis and the origin of dairy starter cultures. Genome Biol. Evol. 2:729-744.

Khanal, S. N., and J. A. Lucey. 2018. Effect of fermentation temperature on the properties of exopolysaccharides and the acid gelation behavior for milk fermented by Streptococcus thermophilus strains DGCC7785 and St-143. J. Dairy Sci. 101:3799-3811.

Kõljalg, U., K. H. Larsson, K. Abarenkov, R. H. Nilsson, I. J. Alexander, U. Eberhardt, S. Erland, K. Hoiland, R. Kjoller, E. Larsson, T. Pennanen, R. Sen, A. F. Taylor, L. Tedersoo, T. Vralstad, and B. M. Ursing. 2005. UNITE: A database providing web-based methods for the molecular identification of ectomycorrhizal fungi New Phytol. 166:1063-1068.

Krzyściak, W., K. K. Pluskwa, A. Jurczak, and D. Koscielniak. 2013 The pathogenicity of the Streptococcus genus. Eur. J. Clin. Microbiol. Infect. Dis. 32:1361-1376.

Li, D., J. Li, F. Zhao, G. Wang, Q. Qin, and Y. Hao. 2016. The influence of fermentation condition on production and molecular mass of EPS produced by Streptococcus thermophilus 05-34 in milkbased medium. Food Chem. 197(Pt A):367-372.

Lusk, T. S., A. R. Ottesen, J. R. White, M. W. Allard, E. W. Brown, and J. A. Kase. 2012. Characterization of microflora in Latin-style cheeses by next-generation sequencing technology. BMC Microbiol. 12:254.

Markiewicz-Keszycka, M., G. Czyżak-Runowska, P. Lipińska, and J. Wójtowski. 2013. Fatty acid profile of milk: A review. Bull. Vet. Inst. Pulawy 57:135-139.

Martin, N. H., A. Trmcic, T. H. Hsieh, K. J. Boor, and M. Wiedmann. 2016. The evolving role of coliforms as indicators of unhygienic processing conditions in dairy foods. Front. Microbiol. 7:1549.

Murugesan, S., M. P. Reyes-Mata, K. Nirmalkar, A. Chavez-Carbajal, J. I. Juarez-Hernandez, R. E. Torres-Gomez, A. Pina-Escobedo, O. Maya, C. Hoyo-Vadillo, E. G. Ramos-Ramirez, J. A. SalazarMontoya, and J. Garcia-Mena. 2018. Profiling of bacterial and fungal communities of Mexican cheeses by high throughput DNA sequencing. Food Res. Int. 113:371-381.

Perez, F., and R. A. Bonomo. 2019. Carbapenem-resistant Enterobacteriaceae: Global action required. Lancet Infect. Dis. 19:561-562.

Pruesse, E., C. Quast, K. Knittel, B. M. Fuchs, W. Ludwig, J. Peplies, and F. O. Glockner. 2007. SILVA: A comprehensive online resource for quality checked and aligned ribosomal RNA sequence data compatible with ARB. Nucleic Acids Res. 35:7188-7196.

Rong, J., H. Zheng, M. Liu, X. Hu, T. Wang, X. Zhang, F. Jin, and L. Wang. 2015. Probiotic and anti-inflammatory attributes of an isolate Lactobacillus helveticus NS8 from Mongolian fermented koumiss. BMC Microbiol. 15:196.

Shuangquan, W. T. Gao, Nairuga, N. Wu, Yamei, Hasiqimuge, and Sarula.. 2018. The production process and nutritional characteristics of Mongolian traditional fermented vrum. Food Res. Devel. 39:154-159. [In Chinese].

Slattery, L., J. O'Callaghan, G. F. Fitzgerald, T. Beresford, and R. P. Ross. 2010. Invited review: Lactobacillus helveticus - A thermophilic dairy starter related to gut bacteria. J. Dairy Sci. 93:44354454 .

Sun, Z., W. Liu, Q. Bao, J. Zhang, Q. Hou, L. Kwok, T. Sun, and H. Zhang. 2014. Investigation of bacterial and fungal diversity in tarag using high-throughput sequencing. J. Dairy Sci. 97:60856096.

Sun, Z., W. Liu, Y. Song, H. Xu, J. Yu, M. Bilige, H. Zhang, and Y. Chen. 2015. Population structure of Lactobacillus helveticus isolates from naturally fermented dairy products based on multilocus sequence typing. J. Dairy Sci. 98:2962-2972. 
Tian, L., S. J. Perot, S. Hon, J. Zhou, X. Liang, J. T. Bouvier, A. M. Guss, D. G. Olson, and L. R. Lynd. 2017. Enhanced ethanol formation by Clostridium thermocellum via pyruvate decarboxylase. Microb. Cell Fact. 16:171.

Valderrama, M. J., M. I. de Siloniz, P. Gonzalo, and J. M. Peinado. 1999. A differential medium for the isolation of Kluyveromyces marxianus and Kluyveromyces lactis from dairy products. J. Food Prot. 62:189-193.

Wang, J., T. Wu, X. Fang, W. Min, and Z. Yang. 2018a. Characterization and immunomodulatory activity of an exopolysaccharide produced by Lactobacillus plantarum JLK0142 isolated from fermented dairy tofu. Int. J. Biol. Macromol. 115:985-993.

Wang, Q., G. M. Garrity, J. M. Tiedje, and J. R. Cole. 2007. Naive Bayesian classifier for rapid assignment of rRNA sequences into the new bacterial taxonomy. Appl. Environ. Microbiol. 73:5261-5267.

Wang, X., J. Xiao, Y. Jia, Y. Pan, and Y. Wang. 2018b. Lactobacillus kefiranofaciens, the sole dominant and stable bacterial species, exhibits distinct morphotypes upon colonization in Tibetan kefir grains. Heliyon 4:e00649.

Xiao, F. 2013. Nutrition analysis of wrum and fermented milk curd in Xilingol league of Inner Mongolia. China Dairy Industry 44:57-60. [In Chinese].

Xing, Z., W. Tang, W. Geng, Y. Zheng, and Y. Wang. 2017. In vitro and in vivo evaluation of the probiotic attributes of Lactobacillus kefiranofaciens XL10 isolated from Tibetan kefir grain. Appl. Microbiol. Biotechnol. 101:2467-2477.

Yu, J., W. H. Wang, B. L. Menghe, M. T. Jiri, H. M. Wang, W. J. Liu, Q. H. Bao, Q. Lu, J. C. Zhang, F. Wang, H. Y. Xu, T. S. Sun, and H. P. Zhang. 2011. Diversity of lactic acid bacteria associated with traditional fermented dairy products in Mongolia. J. Dairy Sci. 94:3229-3241.

Zhao, H. X., and Y. B. Li. 2007. Processing and nutrition analysis of Wrum and fermented milk curd in Inner Mongolia. Food Sci. Technol. 12:127-129. (in Chinese). 